\title{
The Extended Superomedial Pedicle: Advancing Mammaplasty Techniques*
}

\author{
Albert Losken ${ }^{\#}$, Christopher D. Funderburk, Claire Duggal \\ Division of Plastic and Reconstructive Surgery, Emory Unversity, Atlanta, USA. \\ Email: \#alosken@emory.edu
}

Received April 14 ${ }^{\text {th }}$ 2012; revised May 16 ${ }^{\text {th }}, 2012$; accepted June 20 ${ }^{\text {th }}, 2012$

\begin{abstract}
Many variations can be applied to traditional mammoplasty techniques to improve outcomes in certain situations. The purpose of this report was to demonstrate the indications and benefits of mammoplasty autoaugmentation using an extended superomedial pedicle. All patients who underwent transfer of an extended superopedicleto other parts of the breast for autoaugmentation were included. Indications were determined and outcomes were assessed. Forty-eight patients were included in the series. The average follow-up was 2.1 years. Indications were categorized into reconstruction of a partial mastectomy defect (oncoplastic group, $\mathrm{n}=18$ ), upper pole volume in the contralateral mastopexy (implant reconstruction group, $n=9$ ), and volume improvement and suspension (massive weight loss group, $n=21$ ). The overall complication rate was $15 \%(n=7 / 48)$, with a revision rate of $10 \%(5 / 48)$. The extended superomedial pedicle is a reliable and versatile adjunct to regular mastopexy techniques for various indications. It gives us the ability to transfer vascularized tissue from the lower pole to areas that require autoaugmentation.
\end{abstract}

Keywords: Autoaugmentation; Mastopexy; Massive Weight Loss; Oncoplastic

\section{Introduction}

Numerous options exist to mobilize the nipple during breast reduction and mastopexy techniques [1]. The superomedial pedicle is one such option that has gained popularity by Dr Hall Finlay who felt that the superior pedicle based more medially is easier to rotate [2]. This technique is reliable and versatile and can be used for reduction and mastopexy techniques, with predictable results [3]. Although the selected pedicle is important in how it maintains nipple viability, of equal importance is how it can be utilized with the remaining breast tissue to reshape the breast mound. We do occasionally encounter situations where additional parenchyma is required in remote parts of the breast not appropriately filled with the pedicle and remaining breast tissue. The use of local flaps and autoaugmentation techniques has subsequently become popular option to rotate tissue into areas of volume void. These can be taken either from within the breast mound or locally [4-8]. The superomedial pedicle is ideal for an extension type pedicle since the removal of tissue in the lower pole is often desired to allow glandular plication and breast shaping. Glandular tissue is often abundant in the lower pole and can subsequently be ro-

\footnotetext{
*Conflicts of interest: None declared; Ethical approval: Not required.

${ }^{\#}$ Corresponding author.
}

tated on a well-vascularized pedicle to areas where volume is required.

The purpose of this review was to evaluate additional indications and outcomes using the extended superomedial approach along with traditional reduction or mastopexy techniques.

\section{Methods}

A retrospective chart review was performed on all patients at Emory University Hospital from 2003 to 2009 who underwent a breast reduction or mastopexytechniqueby a single surgeon using the superomedial pedicle. For inclusion in this series, the pedicle was extended down to the inframammary fold and rotated to autoaugment other parts of the breast. Patient demographics and risk factors were queried, and the indications and locations for autoaugmentationof the superomedial pedicle were determined. Outcomes were evaluated including complications and the need for revisional surgery.

Technique: The pre-operative markings for an extended superomedial pedicle are essentially the same as for a regular superomedialmastopexy or reduction. The breast meridian is marked, and the vertical pattern is drawn by displacement of the mound. The nipple is incised and the superomedial pedicle is de-epithelialized. The extended portion is taken down to just above the 
level of the inframammary fold (IMF) and this is also deepithelialized. The extended pedicle is transfer to the upper or outer quadrant for partial mastectomy defects (Figures 1 and 2), or to the upper pole for fullness in the weight loss patient (Figures $\mathbf{3}$ and $\mathbf{4}$ ) or to match an implant reconstruction (Figure 5). The medial and lateral pillars are then created and the extended pedicle is lifted off the chest wall. If the pedicle is too large it is trimmed to the appropriate size for the indicated defect. It is released of the chest wall only as much as is required for adequate mobilization. The pedicle is tacked into position and the medial and lateral pillars are plicated. The nipple is then inset and skin closed. Closure is either vertical or standard Wise patterns depending on the size and shape of the breast. Patients with a nipple to notch of greater than $35 \mathrm{~cm}$ are generally not ideal candidates for superomedial techniques and often have sufficient breast tissue to not required autoaugmentation techniques.

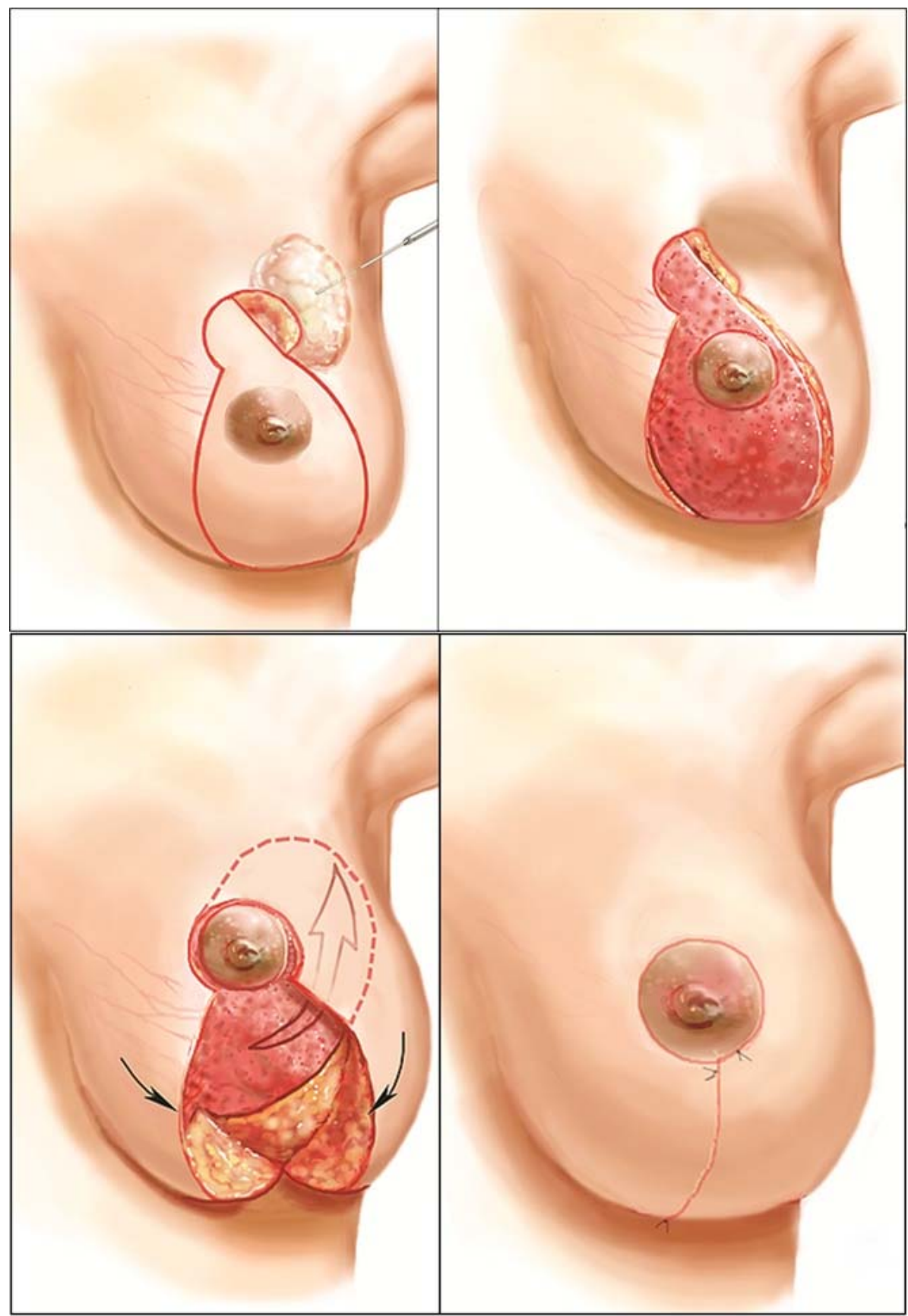

Figure 1. Designing the extended superomedial pedicle for filling a partial mastectomy defect. The extended superomedial pedicle is de-epithelialized to just above the level of the IMF. The extended pedicle is then transferred to the upper or outer quadrant for partial mastectomy defects. 

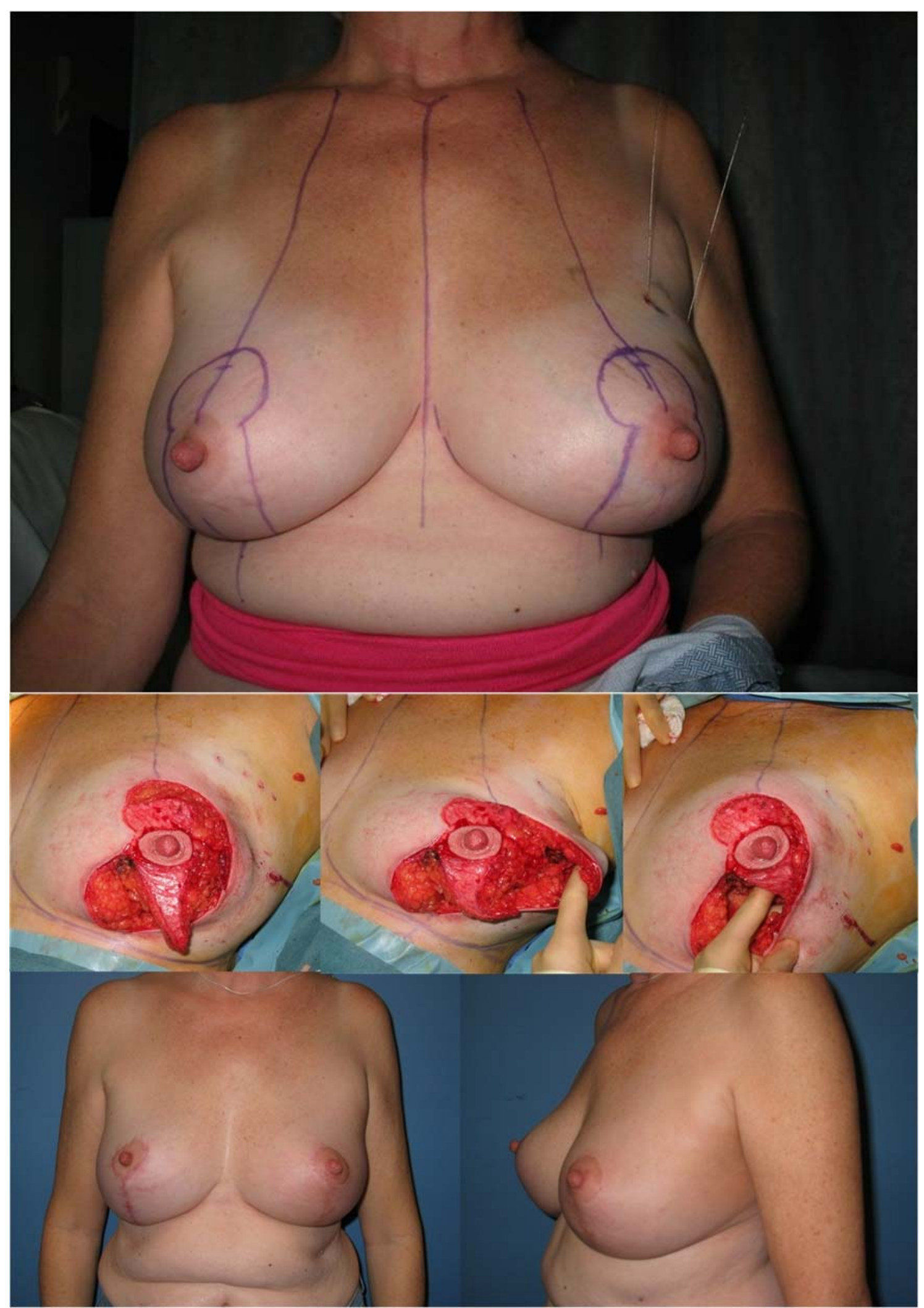

Figure 2. Designing the extended superomedial pedicle for filling a partial mastectomy defect in the upper outer quadrant of the left breast. The pre-operative markings including the breast meridian and a vertical pattern mastopexy are drawn. The extended superomedial pedicle is de-epithelialized to just above the level of the inframammary fold (IMF). The extended pedicle is then transferred to fill the upper outer quadrant defect. Good symmetry is apparent at 6-month follow-up. 


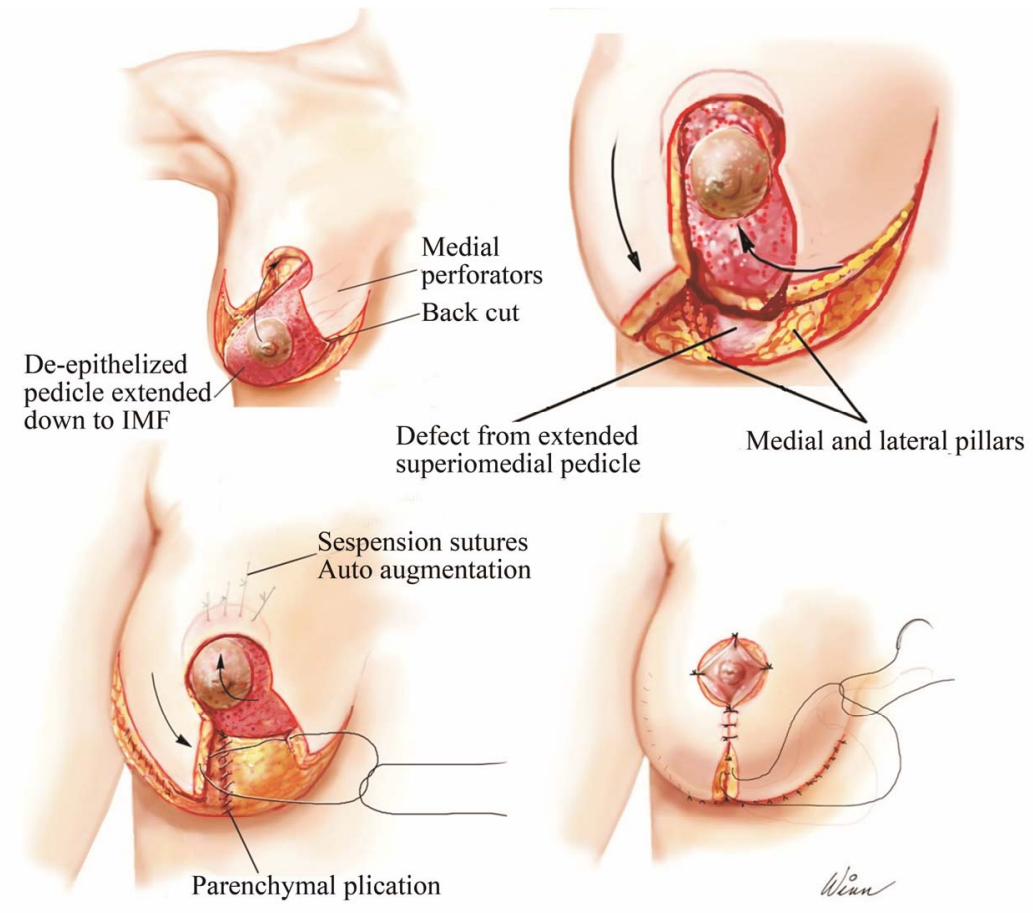

Figure 3. Designing the extended superomedial pedicle for auto-augmentation of the upper pole during mastopexy. The extended superomedial pedicle is de-epithelialized to just about the IMF and rotated superiorly for augmentation. The medial and lateral pillars are created and pilacated and the skin is closed.

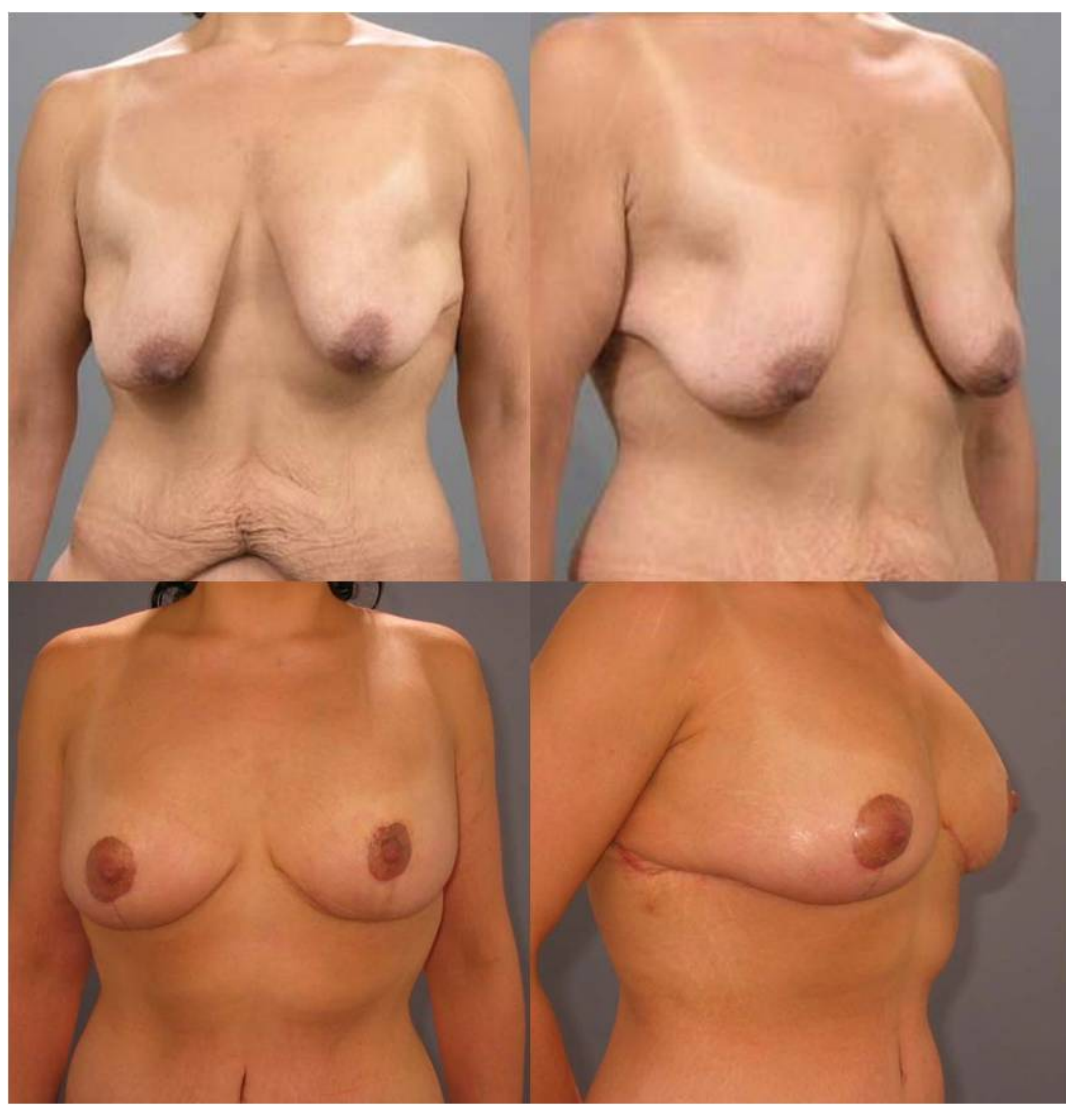

Figure 4. Massive weight loss patient pre-operatively, and post-operatively after mastopexy with extended superomedial autoaugmentation to the upper pole. 


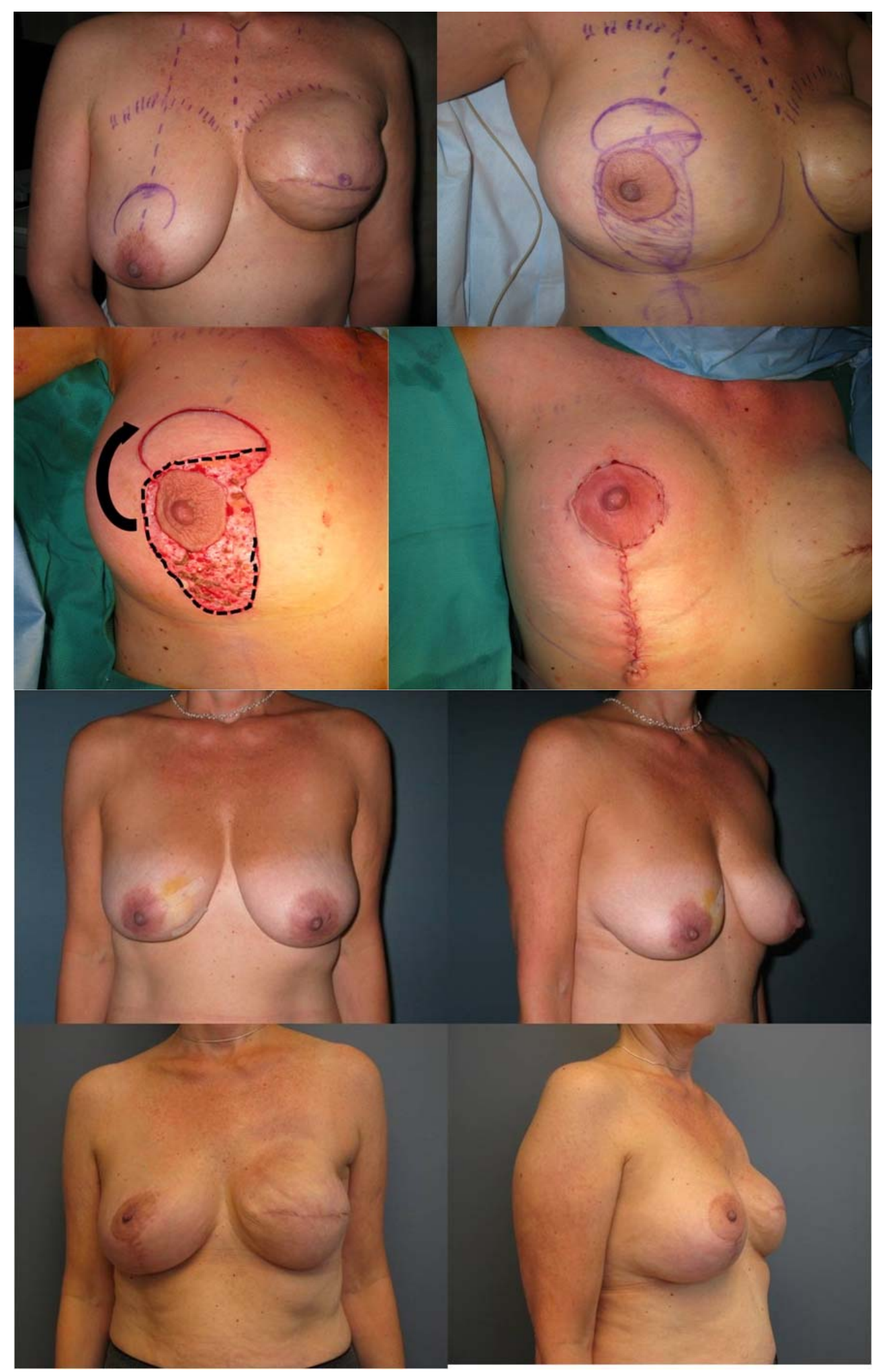

Figure 5. Designing the extended superomedial pedicle for upper pole auto-augmentation to match an implant reconstruction after mastectomy. The extended superomedial pedicle is de-epithelialized to just about the IMF and rotated superiorly for augmentation. Pre-operative views before left mastectomy and reconstruction, and post-operatively after left implant reconstruction with right extended superomedial pedicle for upper pole auto-augmentation. 


\section{Results}

A total of 48 patients had a superomedialdermatoglandular extension pedicle. The indications were divided into oncoplastic $(\mathrm{n}=18)$, symmetry procedure for implant reconstruction $(n=9)$ and massive weight loss $(n=21)$. The average follow up was 2.1 years (range: 2 months - 8 years). Early firmness of the autoaugmentation flap (ede$\mathrm{ma} /$ necrosis) was felt in 4 patients (8\%), however, did resolve within a few months in all patients.

\subsection{Oncoplastic Group}

There were 18 patients in the oncoplastic group who had a superomedial extension pedicle. All patients had a diagnosis of breast cancer, and underwent a lumpectomy, which was reconstructed with an immediate oncoplasticmastopexy or reduction procedure. The lumpectomy defects were in the lateral or upper-outer quadrant in all patients. The average weight of the lumpectomy specimen was 217 grams (range: 36 - 400 grams). In only 2 patients was additional volume resected to complete the reconstruction. Complications occurred in 3 patients (3/ 18), and included cellulitis, and wound dehiscence. Two patients required revisional surgery for recurrence or radiation changes. There were no complications related to the extended dermatoglandular pedicle.

\subsection{Implant Reconstruction Group}

There were 9 patients who had the superomedial extension pedicle for upper pole autoaugmentation as a symmetry procedure along with implant based breast reconstruction. All patients had a history of breast cancer. Additional tissue was resected in one patient (100 grams). There were no complications attributed to the symmetry procedure, and one patient (1/9) required a mastopexy revision for symmetry.

\subsection{Massive Weight Loss Group}

There were 21 patients in the massive weight loss category whohad autoaugmentaion of the upper pole using an extended superomedial pedicle. The average weight loss in this group was 54 kilograms (range 36 - 84 kilograms). Complications occurred in 4 patients $(19 \%, n=4 / 21)$ and included minor wound dehiscence. Two patients (10\%) did require a revision mastopexy for recurrent ptosis.

\section{Discussion}

The extended superomedial technique is indicated whenever autoaugmentation is required using vascularized breast tissue to fill defects or provide additional volume in certain locations within the breast mound. Variousautoaugmentation techniques have been proposed in the literature, mainly in relation to management of the massive weight loss breast [4-11]. Hamdi et al. described using excess lateral tissue based on the lateral intercostal artery perforators to autoaugment volume depleted breasts [6]. Akyurek et al. later suggested a modification of this technique to include a pectoralis muscle sling to prevent long termpseudoptosis [12]. The benefits of using the superomedialpedical are that it is a well-vascularized pedicle and has an arc of rotation that is ideal for transfer to the lateral or upper breast region without the need for additional dissection and a donor site. Volume is taken from the lower pole where it is often excessive, and transferred to the other locations where it is often deficient. The superomedial technique also allows plication of the medial and lateral pillars with the benefits of a vertical mammoplasty technique [12]. Kim et al. recently showed minimal complications and high patient satisfaction using a superior pedicle autoaugmentationmastopexy technique [13]. Since this technique does not bring in tissue from outside the breast, patients who have insufficient breast volume and still desire upper pole fullness will require implant augmentation or alternative techniques. Calvert et al. also found high patient satisfaction using thesuperomedial pedicle mastopexyautoaugmentationtechnique in combination with an implant [14].

In addition to being a useful adjunct to cosmetic mastopexy techniques in women who wish to have upper pole fullness, the indications in this series fell into three major groups. Reconstruction of the upper outer or lateral partial mastectomy defect in women with moderate sized breasts can be challenging without local flaps. The ability to mobilize residual breast tissue for this purpose is ideal and allows filling of the lumpectomy defect with vascularized tissue prior to radiation therapy subsequently preserving shape. This technique can fill a partial mastectomy defect in any quadrant except one in the superomedial location. If a similar procedure is done on the opposite side, symmetry can also be preserved. This approach relies on there being sufficient breast tissue following resection to keep the nipple areaolar complex (NAC) alive and reshape the mound. Occasionally the defect is such that breast tissue in that location is not enough to fill the defect. The nipple pedicle is often limited by how it can be rotated since nipple needs to be in a particular location on the breast mound. This is where autoaugmentation techniques are used to fill the defect. The extended superomedial pedicle will allow rotation of the NAC into the desired location, as well as repositioning lower pole breast tissue into the defect without a donor site.

Reshaping the long and atrophic massive weight loss breast is often a challenge, and repositioning lower pole tissue for upper autoaugmentation is beneficial for numerous reasons [4]. The extended superomedial pedicle 
can be used in a rotation-advancement fashion to fill the upper pole volume deficiency, narrow the base of the breast, and tighten the IMF. In addition to autoaugmentation, the extended pedicle can also be used to suspend the mound superiorly. Parenchymal reshaping with pillar plication will also allow redraping of the skin over the mound, in an attempt to minimize recurrent ptosis. The revision rate however was still around $10 \%$ in the MWL patient. The final group of patients where this approach was beneficial was as a symmetry procedure to match an implant reconstruction. Over autoaugmentation of the upper pole in the contralateral breast will make matching a unilateral implant reconstruction closer. This provides the desired upper pole fullness to match the implant reconstruction when the size difference is not enough to warrant contralateral implant augmentation. This autoaugmentation technique will often give the appearance of an augmented breast improving overall symmetry.

The superomedial technique has been shown to be both safe and reliable with an acceptable complication rate $[3,13]$. Appropriate flap design with maximizing blood flow will limit the incidence of complications such as tip necrosis. This includes keeping the pedicle base wide enough, and maintaining as many perforators to the chest wall as possible. If the end of the flap appears poorly perfused, it should be discarded. Long term retraction and shape distortion was not observed in our series. The massive weight loss patients carry their usual complication risks such as recurrent ptosis due to the inelastic skin and have a higher incidence of recurrent ptosis and revision. The breast cancer patients are at increased risk of infection and wound healing problems especially in the setting of chemotherapy and radiation therapy. While the extended pedicle will fill the lumpectomy defect and minimizes the potential for a deformity, the risk of radiation fibrosis to the skin flaps does still exist. We recognize the limitations of this review in that it is a relatively small series without any comparison groups, however, was intended more to be a presentation one autoaugmentation option with various indications.

\section{Conclusion}

The extended superomedial pedicle has become a useful carrier for additional dermatoglandular tissue because of its reliable vascularity and easy arc of rotation to the outer or upper quadrant of the breast. It has proven to be a safe and reliable option without major morbidities, and is an easy addition to the superomedial procedure. The learning curve is minimal, and once comfortable with this approach it can be a versatile addition to cosmetic and reconstructive breast procedures. The benefits of removing tissue from the lower pole are numerous, and this tissue can be used to treat areas of volume void in other parts of the breast. Autoaugmentation techniques will likely continue to gain popularity as our indications for their use broaden, and our understanding of the vascular anatomy improves.

\section{REFERENCES}

[1] R. J. Rohrich, A. A. Gosman, S. A. Brown and J. Reisch, "Mastopexy Preferences: A Survey of Board-Certified Plastic Surgeons,” Plastic \& Reconstructive Surgery, Vol. 118, No. 7, 2006, pp. 1631-1638. doi:10.1097/01.prs.0000248397.83578.aa

[2] E. J. Hall-Finlay, “A Simplified Vertical Reduction Mammaplasty: Shortening the Learning Curve,” Plastic \& Reconstructive Surgery, Vol. 104, No. 3, 1999, pp. 748-759. doi:10.1097/00006534-199909030-00020

[3] S. P. Davison, A. N. Mesbahi, I. Ducic, M. Sarcia, J. Dayan and S. L. Spear, "The Versatility of the Superomedial Pedicle with Various Skin Reduction Patterns," Plastic \& Reconstructive Surgery, Vol. 120, No. 6, 2007, pp. 1466-1476. doi:10.1097/01.prs.0000282033.58509.76

[4] A. Losken and D. J. Holtz, "Versatility of the Superomedial Pedicle in Managing the Massive Weight Loss Breast: The Rotation-Advancement Technique," Plastic \& Reconstructive Surgery, Vol. 120, No. 4, 2007, pp. 10601068. doi:10.1097/01.prs.0000278004.24650.e6

[5] S. Kwei, L. Borud and B. T. Lee, "Mastopexy with Autologous Augmentation after Massive Weight Loss: The Intercosal Artery Perforator (ICAP) Flap,” Annals of Plastic Surgery, Vol. 57, No. 4, 2006, pp. 361-365.

doi:10.1097/01.sap.0000222569.59581.d9

[6] M. Hamdi, K. Van Landuyt, P. Blondeel, et al., “Autologous Breast Augmentation with the Lateral Intercostal Artery Perforator Flap in Massive Weight Loss Patients," Journal of Plastic, Reconstructive \& Aesthetic Surgery, Vol. 62, No. 1, 2007, pp. 65-70.

[7] R. J. Zienowicz, "Augmentation Mammaplasty by Reverse Abdominoplasty,” Plastic \& Reconstructive Surgery, Vol. 124, No. 5, 2009, pp. 1662-1672.

[8] A. S. Colwell, D. Daniel and K. H. Breuning, "Mastopexy Techniques after Massive Weight Loss: An Algorithmic Approach and Review of the Literature," Annals of Plastic Surgery, Vol. 63, No. 1, 2009, pp. 28-33. doi:10.1097/SAP.0b013e318188b976

[9] J. P. Rubin and G. Khachi, "Mastopexy after Massive Weight Loss: Dermal Suspension and Selective Auto-Augmentation," Clinics in Plastic Surgery, Vol. 35, No. 1, 2008, pp. 123-129. doi:10.1016/j.cps.2007.08.008

[10] R. Graf, L. R. Reis de Araujo, R. Ripple, et al., "Reduction Mammaplasty and Mastopexy Using the Vertical Scar and Thoracic Wall Flap Technique,” Aesthetic Plastic Surgery, Vol. 27, No. 1, 2003, pp. 6-12.

[11] G. Botti, "Vertical Scar Mammaplasty: Stable Padding of the Superior Pole by Means of Posterioly Based Pedicle Autoprosthesis," Aesthetic Surgery Journal, Vol. 19, No. 2, 1999, pp. 116-123. doi:10.1053/aq.1999.v19.97039

[12] M. Lejour, "Vertical Mammaplasty and Liposuction of the Breast," Plastic \& Reconstructive Surgery, Vol. 94, No. 1, 1994, p. 100. 
doi:10.1097/00006534-199407000-00010

[13] M. Akyurek, "Vertical Mastopexy and Lateral Intercostal Artery Perforator (LICAP) Flap with Pectoralis Muscle Sling for Autologous Tissue Breast Augmentation in the Bariatric Patient,” Annals of Plastic Surgery, Vol. 66, No. 1, 2011, pp. 29-35. doi:10.1097/SAP.0b013e3181d6e28a
[14] P. Kim, K. Kim and L. Casas, "Superior Pedicle Autoaugmentation Mastopexy: A Review of 34 Consecutive Patients,” Aesthetic Surgery Journal, Vol. 30, No. 2, 2010, pp. 201-210. doi:10.1177/1090820X10366009 